Retraction

\title{
Retracted: Effect of Salinity on Biomass Yield and Physiological and Stem-Root Anatomical Characteristics of Purslane (Portulaca oleracea L.) Accessions
}

\author{
BioMed Research International \\ Received 22 July 2019; Accepted 22 July 2019; Published 8 August 2019 \\ Copyright ( 2019 BioMed Research International. This is an open access article distributed under the Creative Commons \\ Attribution License, which permits unrestricted use, distribution, and reproduction in any medium, provided the original work is \\ properly cited.
}

The article "Effect of Salinity on Biomass Yield and Physiological and Stem-Root Anatomical Characteristics of Purslane (Portulaca oleracea L.) Accessions" [1] has been retracted as it was found to contain duplicated figures and text similarity. Comments on PubPeer noted concerns with Figures 8, 9, and 11 [2]. In Figure 8, the $0 \mathrm{dS} \mathrm{m}^{-1}$ panel is identical to the $8 \mathrm{dS}$ $\mathrm{m}^{-1}$ panel, but with a lower contrast. In Figure 9, the five panels are identical, but rotated or magnified.

In addition, we found that the $8 \mathrm{dS} \mathrm{m}^{-1}$ panel in Figure 10 is identical to the $32 \mathrm{dS} \mathrm{m}^{-1}$ panel in Figure 12 . These concerns are highlighted in the Supplementary File (available here).

We also found a similar article published in Biological Research by the same group of authors [3]. The article's full details are as follows: Md. Amirul Alam, Abdul Shukor Juraimi, M. Y. Rafii, Azizah Abdul Hamid, Farzad Aslani and M. A. Hakim, "Salinity-induced changes in the morphology and major mineral nutrient composition of purslane (Portulaca oleracea L.) accessions," Biological Research, 2016 49:24, https://doi.org/10.1186/s40659-016-0084-5. We sought the authors' clarification and they stated they have lost the data.

\section{Supplementary Materials}

The file includes the problem figures that were spotted by Hindawi and in PubPeer Comments. (Supplementary Materials)

\section{References}

[1] M. A. Alam, A. S. Juraimi, M. Y. Rafii, and A. Abdul Hamid, "Effect of salinity on biomass yield and physiological and stemroot anatomical characteristics of purslane (Portulaca oleracea
L.) accessions," BioMed Research International, vol. 2015, Article ID 105695, 15 pages, 2015.

[2] "Effect of salinity on biomass yield and physiological and stemroot anatomical characteristics of purslane (Portulaca oleracea L.) accessions," 2018, https://www.pubpeer.com/publications/ 8F7CD0A0167BBCF04AD681B54CC848.

[3] M. A. Alam, A. S. Juraimi, M. Y. Rafii, A. A. Hamid, F. Aslani, and M. A. Hakim, "Salinity-induced changes in the morphology and major mineral nutrient composition of purslane (Portulaca oleracea L.) accessions," Biological Research, vol. 49, article no. 24, 2016. 